\title{
Inhibiting the reproduction of COVID-19-causing SARS-CoV-2 through perturbations in human cell metabolic network
}

\author{
hadrien delattre ${ }^{1}$, Kalesh Sasidharan ${ }^{2}$, and orkun soyer ${ }^{1}$ \\ ${ }^{1}$ University of Warwick \\ ${ }^{2}$ Affiliation not available
}

May 12, 2020

\begin{abstract}
Viruses, including the recent, COVID-19-causing SARS-CoV-2 rely on their host for re-production. Here, we made use of genomic and structural information from SARS-CoV-2 and related viruses to create a biomass function capturing the stoichiometric amino and nucleic acid requirements of SARS-CoV-2. By incorporating this function into a stoichiometric metabolic model of the human cell and applying metabolic flux balance analysis, we identified host-based metabolic perturbations inhibiting SARS-CoV-2 reproduction. Our results highlight reactions in the central metabolism, as well as amino acid and nucleotide biosynthesis pathways. By incorporating host cellular maintenance into the model based on available protein expression data from human lung cells, we find that only few of these metabolic perturbations are able to selectively inhibit virus reproduction without significantly affecting host maintenance. Key reactions that are able to do so are found in metabolic junctions in amino acid biosynthesis pathways and in mitochondrial metabolite shuttles. We note that some of the catalysing enzymes of such reactions have demonstrated interactions with existing drugs, which can be used for experimental testing of the presented predictions. The developed computational approach offers a platform for rapid, experimentally testable generation of drug predictions against existing and emerging viruses based on their biomass requirements and their host's metabolism.
\end{abstract}

\section{INTRODUCTION}

One of the crucial steps in the virus life cycle is the synthesis of the virus particles within the host cell. This includes the synthesis of viral structural proteins and new genomic material. For these processes, all viruses are fully dependent on their host for the required energy (Mahmoudabadi 2017) and building blocks (Berzin 1974; Waldbauer 2019). This dependency is evidenced by experimental findings showing significant metabolic flux alterations in host cells upon infection (Maynard 2010a; Yu 2011). Systems level metabolic studies have particularly highlighted changes in glucose uptake and glycolysis (El-Bacha 2004; Munger 2006), which might be related to an increased demand for biosynthetic precursors as viral production becomes the dominant process within infected cells (Berzin 1974).

The observation of virus synthesis dominating the metabolism and physiology of infected cells suggests that it might be possible to manipulate the latter to control the former (Maynard 2010a; Ikeda 2007). Indeed, several of the existing antivirals such as Ribavirin, Remdesivir, and Gemcitabine are nucleoside analogs that target metabolic enzymes in the nucleotide biosynthesis pathways and are thought to function through their impact on free nucleotide pools in the cell (Wang 2011; Leyssen 2008). An even more specific metabolic approach to inhibit virus production was demonstrated in the case of human cytomegalovirus. For this virus, metabolic analyses highlighted a shifting of metabolic fluxes within central carbon metabolism and fatty acid biosynthesis pathways during infection (Munger 2008). It was predicted that these flux changes could be blocked by perturbation of specific enzymes, which were then targeted with available inhibitors and resulted 
in reduced virus production (Munger 2008). Systematic analysis of gene knockout effects on infection of bacteria with phage also identified many metabolic genes associated with central carbon metabolism and substrate transport (Maynard 2010b), leading to the proposition of utilising host metabolic engineering to modulate viral production (Maynard 2010a). Such metabolic control has been explored in virus-based bioproduction using insect cells, where alterations in the culture media allowed alteration of metabolic fluxes and production levels (Carinhas 2010).

These experimental findings show that viral biomass synthesis causes significant metabolic flux changes in host cells and that metabolic perturbations can directly alter virus reproduction. Thus, system-level metabolic models could be utilised to predict what types of metabolic alterations can cause what kinds of impact on virus reproduction. While modelling of virus reproduction in host cells has mostly taken a kinetic approach, focusing on translation and transcription processes (Endy 1997; You 2002, Yin 2018), it has been possible to combine such kinetic models with genome-scale metabolic models to account for both host and virus biomass (Jain 2009). This allowed predicting the effects of metabolites available in the culture media on the dynamics of the infection process (Birch 2012). In a human cell context, genome-scale metabolic models were utilised to analyse the metabolic impact of infection of macrophages with bacteria or viruses (Bordbar 2010; Aller 2018). One of these studies incorporated viral production into the macrophage metabolic model and predicted specific reaction perturbations that can cause a reduction in viral reproduction (Aller 2018). These predictions correctly identified enzyme targets of the aforementioned antiviral drugs in nucleotide pathways and highlighted new target enzymes (Aller 2018). Such findings from the virus-host metabolic modelling aligns with the observations that genome-scale metabolic models can provide a comprehensive stoichiometric catalogue of possible biochemical conversions in a cell (Thiele 2013; Swainston 2016) and that can generate useful qualitative predictions on the impact of environmental or genetic alterations on the cellular metabolic flux distributions (Edwards 2000; Segré 2002; Papp 2004).

Motivated by the qualitative predictive power of stoichiometric metabolic models and flux analysis, we apply it here to simulate the production of SARS-CoV-2 virus particles as part of the host metabolism and predict metabolic inhibitions against this virus. We extend the available human genome-scale metabolic model with a viral biomass reaction, estimated using structural information available from SARS-CoV-2 and related viruses. By optimising flux distributions in this model for viral reproduction, we were able to predict reactions that can inhibit this process. We explored the possible impact of these predicted inhibitions on the host metabolism itself, using an estimation of host cell maintenance in human lung cells based on available protein expression data. In addition, we have explored experimental feasibility of implementing the predicted metabolic perturbations using available drug and inhibitor information on metabolic enzymes. Our results indicate that individual and double perturbation of several metabolic reactions from central metabolic pathways can inhibit SARS-CoV-2 reproduction production in general and some of these can do so selectively without affecting normal metabolic functions of the host. We highlight these reactions as experimentally testable drug targets for inhibiting SARS-CoV-2 reproduction in human lung cells and provide details of the implemented computational approach for further development.

\section{RESULTS AND DISCUSSION}

To simulate the production of SARS-CoV-2 virus particles in a human cell we utilised an existing, communitydeveloped human genome scale model known as RECON 2.2 (Thiele 2013; Swainston 2016) (see Methods ). This model represents the most comprehensive catalogue of metabolic reactions found in human cells, with many of its reactions associated with known genes (Swainston 2016). Within this model, we implement a pseudo reaction representing the production of SARS-CoV-2 viral particles from biosynthetic precursors (see Figure 1A and Methods ). The construction of this pseudo reaction is based on available structural information on the virus including its use of proteins for viral packaging (Bar-On 2020; Mahmoudabadi 2017; Bárcena 2009; Neuman 2006; 2008; 2011) and its genome sequence. As such, this pseudo biomass reaction accounts for the stoichiometry of nucleic and amino acids required to make a complete virus and associated energetic 
costs. This analysis highlights that leucine and alanine are the most utilised amino acids in SARS-CoV-2 proteins and adenosine- and uridine-triphosphate are the more common nucleotides in its RNA (Figure 1B).

Metabolic fluxes supporting SARS-CoV-2 production in a human cell are primarily in central carbon metabolism. By incorporating the SARS-CoV-2 viral biomass function into the human metabolic model and assuming a minimal media composition (see Methods ) we predict a metabolic flux distribution for optimal virus production in a human cell (Figure 1C). We then evaluated the flux variability allowed in each reaction of the model, while maintaining an optimal viral production level (Supplementary File 1). These analyses have shown that reactions which must carry flux for optimal viral biomass production include glycolysis, oxidative phosphorylation, fatty acid oxidation, and specific amino and nucleic acid biosynthesis pathways (Figure 1C andSupplementary File 1). As the optimal flux distributions are related also to the flux limits imposed on uptake reaction fluxes, we also repeated the flux variability analysis with minimal media but using an increased uptake limit and with a rich media that allows all uptake reactions of the model to be active. Increasing uptake limits did not alter the general conclusions about key active pathways sustaining optimal virus production, but resulted in higher uptake fluxes causing additional pathways relating to overflow metabolism to be active (Supplementary File 1). Simulating a rich media resulted in a much lower number of flux-carrying reactions as the cell can obtain several key compounds such as uridine-triphosphate from the media under this scenario (Supplementary File 1). Since, such a rich media is physiologically less realistic, we focus the remaining analysis on the results from simulations using the minimal media.

Inhibiting specific metabolic enzymes and enzyme combinations inhibit SARS-CoV-2 production in human cells. To identify reaction perturbations which, when inhibited, can halt or reduce virus production, we systematically simulated a knock-out of each flux-carrying reaction. Excluding reactions involved in uptake from media, this analysis highlighted 35 reaction knock-outs that can stop virus production and an additional 8 reactions that can reduce it below $80 \%$ of the original (Figure 1C). The former group of reactions tended to be involved in nucleotide and amino acid biosynthesis pathways, while the latter group included reactions primarily in glycolysis and oxidative phosphorylation (Table 1 and Supplementary File 2). Key ones among these reactions are further discussed below.

Considering that it is possible for the effects of single perturbations to be circumvented by re-directing of fluxes, we also explored combined perturbations. We created all possible pairs of flux-carrying reactions according to the flux variability analysis (over 5000 pairs) and simulated the effect of setting their reaction fluxes to zero. This has identified over 400 reaction pairs, co-inhibition of which results in the reduction of virus optima to $80 \%$ or less of the original (Table 1 and Supplementary File 2). Most of these reaction pairs involved one of the 10 single perturbations that were found to reduce virus production to less than $80 \%$ on their own, but pairing them with additional reaction increased their impact. For example, inhibition of glyceraldehyde-3-phosphate dehydrogenase (GAPD) and cytochrome c oxidase (CYOR) individually caused reduction to $62 \%$ and $60 \%$ of original virus production respectively, but combined inhibition of these reactions results in $25 \%$ of original production (Supplementary File 2). Some of these cases of increased effect arises due to co-inhibition of reactions more effectively blocking fluxes into virus biomass precursors. For example, combined blocking of GAPD and CYOR, reactions involved in respiration and glycolysis respectively, results in reduced fluxes into pyruvate and alpha-ketoglutarate (akg), a key intermediary of the tricarboxylic acid (TCA) cycle. Akg is further linked into valine production through the valine:3-methyl-2-oxobutanoate shuttle across the mitochondrial membrane (Figure 2). In the optimal flux distribution for SARS-CoV-2 production, this "valine shuttle" has a high flux and contributes to the production of both valine and multiple other amino acids via mitochondrial glutamate (Figure $2 \mathrm{~b}$ ). Perturbations to both CYOR and GAPD lead to a new flux distribution where the glutamate production in the mitochondrial matrix is sustained through a different metabolic route. The "valine shuttle" that was active in the optimal solution is now non-functional and is instead replaced by a leucine:4-methyl-2-oxopentanoate shuttle carrying a lesser flux. This in turn decreases the production of the amino acids from glutamate, and thus causing a significant decrease in virus biomass production flux (Figure 2).

In the case of simulating the minimal media with higher uptake fluxes, we have also identified pairs of 
completely new enzyme inhibitions, that were not causing any effect on their own (Supplementary File 2). Some of these pairs exert their effects by blocking multiple pathways from a given compound and thereby causing disruption in steady state balances in the model. For example, co-inhibition of citrate synthase (CSm) and several other enzymes such as histidase (HISD) totally prevents flux in SARS-CoV-2 biosynthesis reaction by making impossible the mass balance of protons in the cytosol (see Figure S1).

Metabolic requirements of viral production are different to those arising from host cellular maintenance. In the above discussed analyses, we considered host metabolism as optimised for viral production and evaluated impact of perturbations only on this process. Such metabolic perturbations should also be evaluated for their impact on the normal metabolic functions of uninfected host cells. In previous studies, normal state of metabolism in human cell lines are either represented through a pseudo reaction for cellular maintenance (Bordbar 2010; Thiele 2013) or through consideration of specific metabolic functions such as ATP or lipid production (Gille 2010; Mardinoglu 2014). In the former case, cell maintenance is captured by a generic account of cellular constituents such as lipids, carbohydrates and DNA and a more specific accounting of amino acid usage in protein expression (Thiele 2013; Bordbar 2010;). In RECON2.2, the protein-based component of the maintenance function is calculated from a large collection of human genes' open reading frames (Thiele 2013).

Here, we expanded from this approach and calculated the biomass protein component using available protein expression data for lung cells from the Human Protein Atlas project (Uhlén 2015) (see Methods ). Comparing the resulting human lung cell maintenance function to the SARS-CoV-2 biomass, in terms of the building block stoichiometries, revealed differences in relative amino and nucleic acid usage (Figure 3 and Supplementary File 3). Compared to the host, there was particularly higher relative usage of phenylalanine, isoleucine, asparagine, threonine, tryptophan, and tyrosine in the virus and particularly lower relative usage of glutamate, histidine, methionine, and proline (Figure 3). Accordingly, the optimisation of the model using the host metabolic maintenance results in a different metabolic flux distribution compared to viral production (Supplementary File 4). The differences, however, were rather limited from the perspective of fluxes supporting SARS-CoV-2 production; out of all reactions that must carry a flux to sustain a virus optimal state, almost all were also required to carry flux to sustain a host optimal state (Supplementary File 4).

Flux control can ensure selective reduction in viral production. Given the above finding that the same reactions carrying flux for SARS-CoV-2 production also carry flux for host metabolic maintenance, we re-analysed the effects of enzyme perturbations on both virus and the host. We found that many of the previously identified single perturbations limiting virus production also limited significantly the host metabolic maintenance, with only one single perturbation - that involving CYOR - showing more than $5 \%$ difference in its impact on virus vs. the host (Supplementary File 5). The same finding prevailed for double perturbations. The only pairs that displayed $5 \%$ or more difference in their effects on virus vs. host are those involving CYOR paired with other enzymes (Supplementary File 5).

The limited differential impact of full inhibition of enzymes made us postulate that more refined perturbations could provide a better strategy to just impact SARS-CoV-2 production without affecting the host. In particular, given the differences in optimal metabolic fluxes between virus production and host maintenance states, we argued that there might be flux values for some reaction that are compatible with only one of these states. To explore this possibility, we systematically analysed the flux variability of each reaction given either the optimisation of host maintenance or virus production. This has allowed us to see if any of the reactions would have flux regimes that are only compatible with the optimal host maintenance but not with optimal virus production and then 'enforce' such flux regimes on the model. This approach allowed us to identify few double reaction perturbations that are fully selective on their effect and solely reduce virus production without causing any impact on the host (see Table 1). All of these involved threonine deaminase (THRD) and caused up to $17 \%$ reduction in SARS-CoV-2 production (Table 1).

The flux enforcement approach creates further constraints on how the metabolic fluxes in the system can be balanced at steady state. In the case of flux enforcements involving THRD this reduced set seems to leave only certain flux distributions to be possible that are specifically less optimal for the virus' biomass 
biosynthesis, but still optimal for host maintenance function to be fulfilled. Expected from this, we found that the effective flux enforcements relate to compositional differences between the viral biomass and host maintenance functions, as illustrated in Figure 4 for the case of the enforcement of THRD and succinate dehydrogenase (SUCD1m). In the optimal flux distribution for SARS-CoV-2 production in the unmodified model, threonine is obtained from the medium through a threonine:leucine shuttle, with both of these amino acids being in relatively similar demand between host and virus requirements. In the case of THRD and SUCD1m fluxes enforced to specific ranges, threonine is instead obtained through a threonine:isoleucine shuttle and is now further intertwined with the arginine:isoleucine shuttle (Figure 4). This shift in fluxes happens because the flux enforcement of SUCD1m, a reaction of the TCA cycle, decreases the flux of the previously observed valine shuttle (see Figure 2) and increases the flux of alternative mitochondrial shuttle reactions including one involving leucine (see Figure 4). The reduced availability of leucine causes the utilisation of isoleucine and arginine for threonine, thereby creating a trade-off among these amino acids. Crucially, arginine is the highest and isoleucine the third highest differentially demanded amino acid when comparing virus vs. host requirements (Figure 3). This is why the described trade-off situation among these amino acids and threonine, caused by the flux enforcement, differentially impacts the SARS-CoV-2 biomass production more than it effects host maintenance.

Current metabolic drugs exist that could target predicted reactions to inhibit production of SARS-CoV-2. The metabolic approach employed here allowed prediction of several reactions and reaction combinations that could limit SARS-CoV-2 production in human cells in general and differentially in human lung cells. The most significant of these are re-summarised in Table 1 as those reactions, the perturbation of which, can reduce virus production below $80 \%$ of the original. For these reactions, we evaluated their associated enzymes in the light of existing, approved drugs using the available small molecule inhibitor and drug database DrugBank (Wishart 2018). We found several existing drugs that could inhibit some of the predicted reactions including those targeting enolase (ENO), phosphoglycerate mutase (PGM), and SUCD1m (Table 2). These drugs could be used as a starting point to experimentally test the predictions made here, using in vitroassays. In addition to these identified small molecule inhibitors, we note that it might also be possible to achieve development of de novo metabolic gene knock-out approaches using recent CRISPR and RNA silencing approaches.

\section{CONCLUSIONS}

Here, we have created a stoichiometric biomass function for the COVID-19-causing SARS-CoV-2 virus and incorporated this into a human lung cell genome scale metabolic model. The viral biomass function highlights the key building blocks required to synthesize a SARS-CoV-2 virus and its simulation within the human metabolic model enables predicting optimal flux distributions in the host for sustaining either SARSCoV-2 reproduction or host maintenance. We used the latter capability to identify reaction perturbations that can inhibit SARS-CoV-2 reproduction in general or selectively, without inhibiting the host metabolic maintenance. The identified reactions primarily fall onto glycolysis and oxidative phosphorylation pathways, and their connections to amino acid biosynthesis pathways. The latter finding is in line with the additional observation we made here, that the relative stoichiometries of specific amino acids differ in SARS-CoV-2 biomass vs. host cell maintenance estimated using human lung cell protein expression data. Together, these results highlight the possibility of targeting host metabolism for inhibition of SARS-CoV-2 reproduction in human cells in general and in human lung cells specifically.

The predictions presented here are based crucially on the structure of the metabolic model as well as the two key assumptions of the flux balance analysis (FBA); the assumptions of metabolic steady state and the optimality of metabolic fluxes towards a specific metabolic function. On the case of the model structure, the RECON2.2 model we used here presents the most-comprehensive and up-to-date curated human genome scale model (Thiele 2013; Swainston 2016). This model contains confidence levels for most included reactions and associated gene information, which can be improved by future studies updating the model structure, an 
area of active development for genome-scale models (Chindelevitch 2015). In the case of the key assumptions of FBA (Schuster 2008; Raman 2009), these are expected not to affect qualitative predictions on which metabolic reactions might be required to carry flux for a given metabolic process or how specific perturbations might impact such processes. For example, FBA-based approaches have been successful in predicting and explaining experimental observations on gene deletion and environmental perturbations in both microbial (Ibarra 2002) and eukaryotic systems (Papp 2004; Gille 2010). In summary, the presented predictions of our FBA-based approach should be taken as starting points for experimental studies and to be improved in a systems biology theory-experiment cycle.

The virus life cycle consists of environmental circulation, infection and subsequent host cell attachment and entry, reproduction within the host cell, and exit for a new round of infection. The presented approach focuses solely on the reproduction within the host cell and the metabolic aspects of that. While this is a limited focus, reproduction in the host cell is a crucial and essential aspect of the virus life cycle. The importance of this stage is highlighted in several studies, which demonstrate that viruses tend to re-program host metabolism for increased viral production (El-Bacha 2004; Munger 2006; Munger 2008; Yu 2011) or encode enzymes that can participate in host metabolic functions (Maynard 2010a). These findings show that metabolic basis of host-virus interaction is crucial for the success of viruses and suggests that such interaction could be under significant evolutionary selection. Emergent viruses, such as SARS-CoV-2, are argued to not be well-adapted to their new host and undergo rapid evolution dictated by host-determined factors (Simmonds 2019). It has been highlighted, for example, that there is a codon usage bias in virus genomes that possibly evolve in time to align with their host (Wong 2010). The presented approach suggests that there might be a similar adaptation of viruses to their host metabolism. We argue that differences in metabolic requirements of a virus vs. its host could be a 'physiological mismatch' that contributes to this evolutionary dynamic. Before metabolic adaptations happen, however, inhibition of the host metabolism might be a possible strategy to selectively inhibit reproduction of emergent viruses in new hosts. Specific host-based metabolic perturbations have already been shown experimentally to be effective against viruses (Munger 2008; Carinhas 2010), while general perturbation of end-points of nucleotide biosynthesis through nucleoside analogs underpins the mode of action of several existing antiviral drugs. The predictions listed here present possible new antiviral targets that are primarily within central carbon metabolism, and in particular in glycolysis and oxidative phosphorylation. There are already several drugs that are shown to interact with the predicted enzymes in this study, opening up the possibility of experimentally testing the presented predictions usingin vitro assays and cell cultures.

In the development of host-based metabolic strategies to inhibit viruses, metabolic modelling, as presented here, can play a useful role. In particular, our approach can be applied relatively rapidly to any host-virus pair both for existing and emerging viruses, and allow generating experimentally testable hypotheses for virus inhibition. This approach can be applied as long as structural and genomic information can be converted into an estimation of biomass composition for the virus and a suitably detailed metabolic model for the host can be constructed. The former process can be enhanced by further databases of viral structural and genomic information, while the latter process would benefit from extending human-focussed efforts such as the human metabolic atlas database (Robinson 2020) to cover also cell lines of common animal hosts.

\section{MATERIALS AND METHODS}

Human genome scale metabolic model and its adaptation to human lung cells. To identify specific host metabolic reactions that can alter viral production, we make use of a generic human cell genome-scale metabolic model that has been previously developed (Thiele 2013) and that has been subsequently curated and improved by the systems biology community (Swainston 2016). This model, referred as RECON2.2, reflects the state-of-the-art in genome scale metabolic model construction applied to human cells and contains over 8000 thousand reactions, many of which have associated gene and protein information (Swainston 2016). This model also contains a pseudo reaction representing generic maintenance costs of a human cell, including 
ATP and precursor stoichiometries for proteins, DNA, RNA, lipids, and carbohydrates. This pseudo biomass maintenance reaction is primarily derived using information from human leukaemia cell lines (Thiele 2013). This generic human cell model represents a consensus human metabolic capacity, and as such, its use in this study allows identification of widest range of possible metabolic reactions which can then be further scrutinized and sifted in a cell specific context.

As discussed in the main text, when identifying metabolic perturbations that can selectively affect viral production without much affecting the host, the specifics of the used host maintenance representation must be cell-specific. To align this representation to a lung cell we have made use of the gene expression data available from the Human Protein Atlas project (Robinson 2020). In particular, we have used the gene expression profile for lung cells available from this project and available protein sequences from ENSEMBL database (Yates 2020) to create a lung-cell specific stoichiometry for amino acids required for protein synthesis. The proportion of each amino acid in the composition of the new maintenance function was determined by converting the protein coding RNA's codons to amino acids, counting their frequency and weighing these with the normalised expression coefficient provided by the Human Protein Atlas project. The energy cost associated with amino acid polymerisation is also accounted for based on the length of the protein sequences, and assuming 4.3 molecules of ATP hydrolysed to ADP per amino acid polymerisation (Quek 2014). The stoichiometric coefficient for each amino acid in the new maintenance function is then scaled so as to represent the same weight as the original protein synthesis reaction in the RECON2.2 model (not accounting for ATP, ADP, $\mathrm{Pi}$ nor $\mathrm{H}_{2} \mathrm{O}$ in the scaling) (see Code Availability). The remaining elements of the maintenance function was retained as in the RECON2.2 model. The final, lung-cell specific maintenance function is provided in Supplementary File 3.

Creation of SARS-CoV-2 virus biomass function. The biomass function for the SARS-CoV-2 is done as in a previous study (Aller 2018) and by accounting for the composition and stoichiometry of proteins and genomic material in the virus. The protein composition and stoichiometry of the virus particle is obtained from electron microscopy and mass spectrometry studies on other coronaviruses, including SARS-CoV (Mahmoudabadi 2017; Barcena 2009; Neuman 2006; Neuman 2008; Neuman 2011). The resulting protein stoichiometry is further checked against recent estimates specific to SARS-CoV-2 (Bar-On 2020). Composition of protein and virus genome sequences are obtained from the National Centre for Biotechnology Information (NCBI) nucleotide database (accession number NC_045512). Energetic costs in form of ATP stoichiometry is calculated as above. The final SARS-CoV-2 biomass function is provided in Supplementary File 3, while the computer code used to calculate is made available (see Code Availability ).

Simulation of the metabolic model. The integrated genome scale metabolic model was simulated using the flux balance analysis (FBA) approach (Bordbar 2014). FBA assumes steady state of metabolic fluxes and implements linear optimisation to find one particular flux distribution across all reactions that can satisfy this assumption and that is optimal under given flux constraints and a certain optimality criterion. Here, we used the standard mathematical implementation of flux balance analysis as described before (Bordbar 2014) and used maximisation of flux through the host maintenance or viral biomass pseudo reactions. All reaction flux constraints are kept as in the original RECON2.2 model except for extracellular transport reactions. The extracellular transport reactions are normally set to carry negative flux to represent uptake of metabolites from the media. In the RECON2.2 model all extracellular transport reactions' minimum flux values are set to $-1000 \mathrm{mmol}[?] \mathrm{gDW}^{-1}[?] \mathrm{h}^{-1}$ (where DW stands for grams of dry weight) to represent a rich media (all exchange reactions allowed to carry flux). We have used here both this approach and additionally implemented a minimal media containing only essential metabolites, carbon and nitrogen source, and oxygen. The identification of the minimal media was achieved using a linear optimisation based algorithmic approach (Senior 2017), where a pseudo currency metabolite is added to all exchange reactions of the model and the flux for the extracellular transport reaction of this pseudo metabolite is systematically altered to identify a minimal set of exchange reactions that can still result in model optimisation. To implement the minimal media the identified extracellular transport reactions' minimum flux values were set to -1000 or to -10 mmol[?]gDW ${ }^{-1}[?] \mathrm{h}^{-1}$, with all other extracellular transport reactions' minimum flux set to zero. The identified media composition is provided as Supplementary File 6 and a computational implementation of the described 
minimal media identification approach is provided in Python (see Code Availability)

TABLES

\begin{tabular}{lllll}
\hline Reaction 1 & Reaction 2 & Virus Optima & Host Optima & Perturbation \\
\hline ATPS4m & - & 75 & 77 & Knock-out \\
ENO & - & 65 & 66 & Knock-out \\
PGM & - & 66 & 67 & Knock-out \\
CYOR & - & 61 & 69 & Knock-out \\
CYOR & GAPD & 25 & 30 & Knock-out \\
CYOR & PUNP3 & 60 & 69 & Knock-out \\
CYOR & ASPTA & 60 & 68 & Knock-out \\
PGK & FTCD & 58 & 59 & Knock-out \\
THRD & CSm & 84 & 100 & Enforcement \\
THRD & SUCD1m & 84 & 100 & Enforcement \\
THRD & MDHm & 84 & 100 & Enforcement \\
THRD & PUNP2 & 84 & 100 & Enforcement \\
\hline
\end{tabular}

Table 1: Selection of reaction perturbations predicted to reduce SARS-CoV-19 biomass production in a human cell with equal or less impact on human lung cell-based metabolic maintenance. Individual or pairs of reaction perturbations are shown, alongside their predicted effects on SARS-CoV-19 and host as percent of optima without any perturbations. For full results see Supplementary Files 2 and 5. Reactions are identified with the short notation used in the RECON2.2 model and their gene and subsystem associations are given in Supplementary File 1. Short notations used are: ATP synthase (ATPS4m), enolase (ENO), phosphoglycerate mutase (PGM), ubiquinol-6 cytochrome c reductase (CYOR), glyceraldehyde-3-phosphate dehydrogenase (GAPD), purine-nucleoside phosphorylase (Guanosine) (PUNP3), aspartate transaminase (ASPTA), phosphoglycerate kinase (PGK), citrate synthase (CSm), formimidoyltransferase cyclodeaminase (FTCD), mitochondrial succinate dehydrogenase (SUCD1m), mitochondrial malate dehydrogenase (MDHm), and purine-nucleoside phosphorylase (Deoxyadenosine) (PUNP2).

\begin{tabular}{lllll}
\hline Reaction & Enzyme & Approved Drug or Inhibitor & Current Use & DrugBank \\
\hline ATPS4m & ATP synthase subunit a & Estradiol & Hormone therapy & DB00783 \\
ATPS4m & ATP synthase subunit c & Bedaquiline & Antimicrobial & DB08903 \\
ENO & Enolase & Sodium fluoride & Antimicrobial & DB09325 \\
PGM/PGK & Phosphoglycerate mutase & Artenimol & Antimicrobial & DB11638 \\
PGM/PGK & Phosphoglycerate mutase & Copper & Diet Supplement & DB09130 \\
FTCD & Formimidoyltransferase cyclodeaminase & Pyridoxal phosphate & Diet Supplement & DB00114 \\
SUCD1m & Succinate dehydrogenase & Ubidecarenone & Diet Supplement & DB09270 \\
\hline
\end{tabular}

Table 2: Existing drugs that are approved or investigated for clinical use and that can target some of the enzymes predicted in this study as causing metabolism-based reduction of SARS-CoV-2 biomass production. Information is collated from DrugBank database (Wishart 2018). The reaction abbreviations are as in Table 1.

\section{FIGURES}


A)

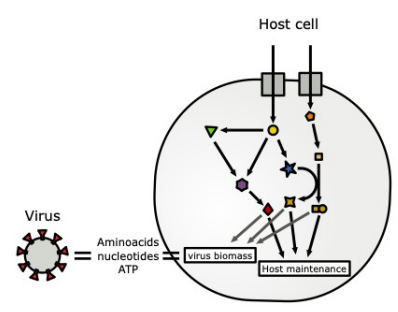

B)

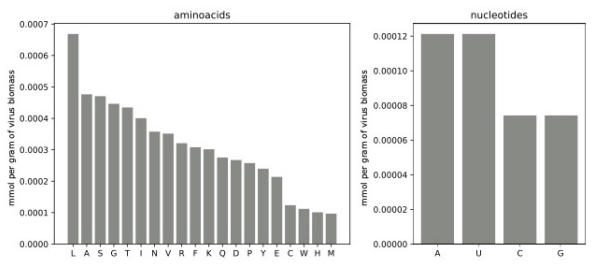

C)

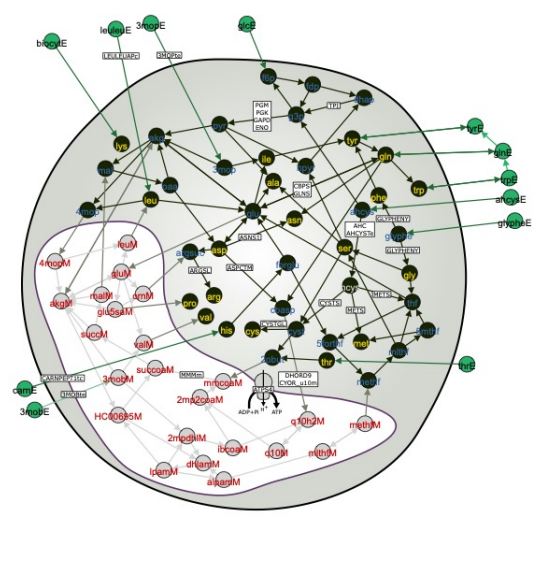

Figure 1. (A) Schematic representation of the integrated host-virus metabolic modelling approach used in this article. The biomass composition of SARS-CoV-2 is estimated as described inMethods and then embedded in the metabolic network model of the host cell. This model is then used to predict the metabolic fluxes supporting virus production and effects of perturbations as described in the main text. (B) Composition of virus biomass in mmol per gram of virus biomass dry weight. The two panels show amino acids and nucleotides as labelled. (C) Graph representation of part of the human cellular metabolic network, focusing on those reactions that are active in virus production under minimal media conditions with uptake fluxes set to $-10 \mathrm{mmol}[?] \mathrm{gDW}^{-1}[?] \mathrm{h}^{-1}$ (see main text andMethods ). The cell is shown as a circle with a grey background, with the mitochondrial matrix shown in white background. Nodes are metabolites and edges are reactions. Edges between two nodes are drawn when at least one reaction connecting those two metabolites carries flux in the optimal flux distribution. The graph shown here is obtained from the full metabolic network by selecting those edges corresponding to the shortest path between metabolites present in the medium and the precursor metabolites to amino acids that contribute to the virus biomass. The paths are found by using the Dijkstra algorithm (Dijkstra 1959) and weighting the edges by the inverse of their flux value. Nodes are coloured by their cellular location; cytosol (black), mitochondrial matrix (grey), external medium (green). Node labels are coloured also according to primarily location, with amino acids and media components labelled in green and yellow respectively. Key reactions discussed in the text and summary results are labelled on their corresponding edges. 
A)
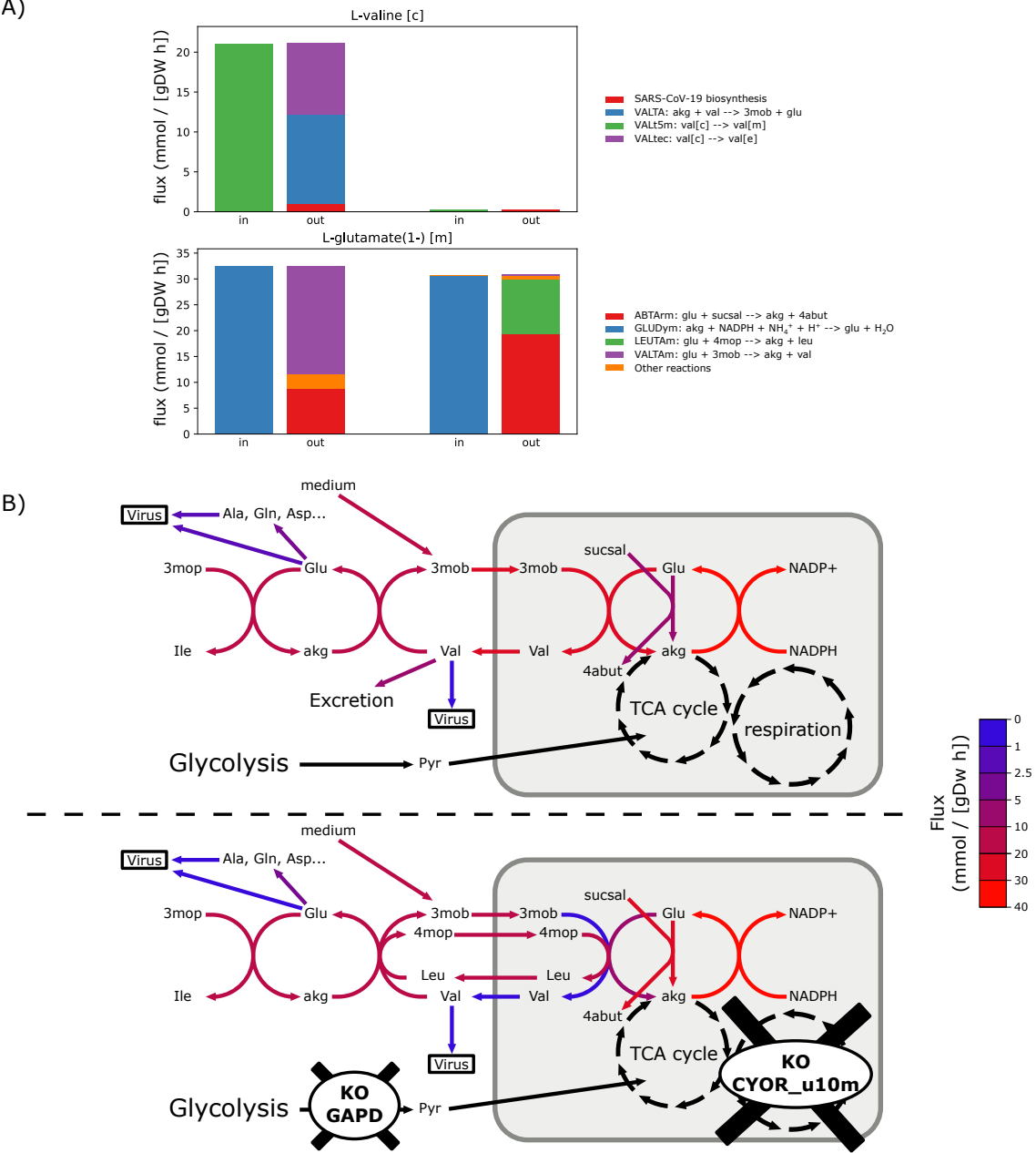

Figure 2. Effect of the double knockout of glyceraldehyde-3-phosphate dehydrogenase (GAPD) and cytochrome $\mathrm{c}$ oxidase (CYOR) on the optimal flux distribution for virus production under minimal media conditions with uptake fluxes set to $-10 \mathrm{mmol}[?] \mathrm{gDW}^{-1}\left[\right.$ ?] $\mathrm{h}^{-1}$. (A)Distribution of the producing ('in') and consuming ('out') fluxes for valine in the cytosol and glutamate in the mitochondrial matrix. For each metabolite, the in and out fluxes have been computed for the unmodified model (left) and for the double knockout of GAPD and CYOR (right). (B) Cartoon representation of the reaction network involving valine and other metabolites, with reaction fluxes from normal and perturbation conditions colour-mapped onto reaction arrows. The upper panel represents the flux distribution in the normal condition, while the lower panel represents flux distribution under perturbation, i.e. when both GAPD and CYOR are knocked out. The light grey rectangle represents the mitochondrial compartment. Metabolite notations used are: 3-methyl-2-oxobutanoate (3mob), 4-methyl-2-oxopentanoate (4mop), alpha-keto-glutarate (akg), 4-oxobutanoate (sucsal), gamma-aminobutyric acid (4abut). 

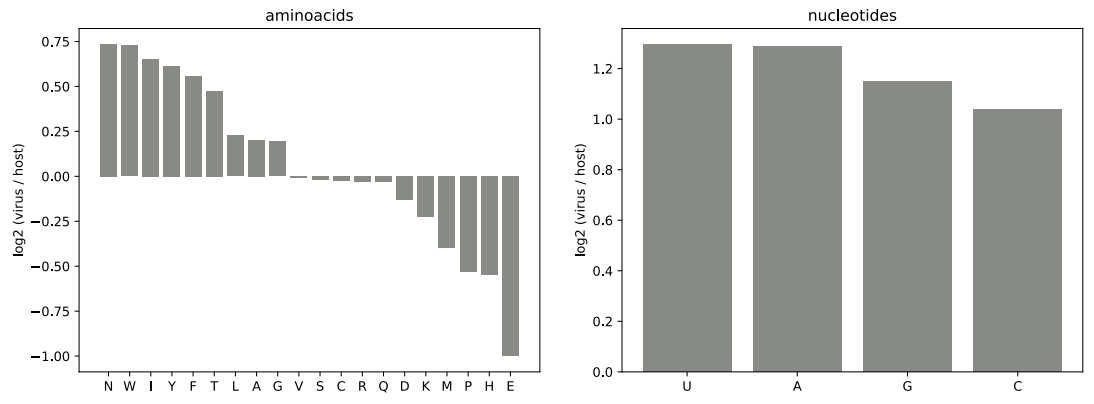

Figure 3. Compositional comparison of SARS-CoV-2 biomass and host maintenance based on human lung cell protein expression. The values on the Y-axis of both panels are the base 2 logarithms of the ratio of the proportion of each metabolite (amino acid or nucleotide) in the virus biomass divided by its proportion in the host maintenance function. The proportion of each metabolite is its stoichiometric coefficient in the corresponding reaction (virus biomass or host maintenance) in mmol[?]gDW-1, divided by the sum of all stoichiometric coefficients in that reaction.
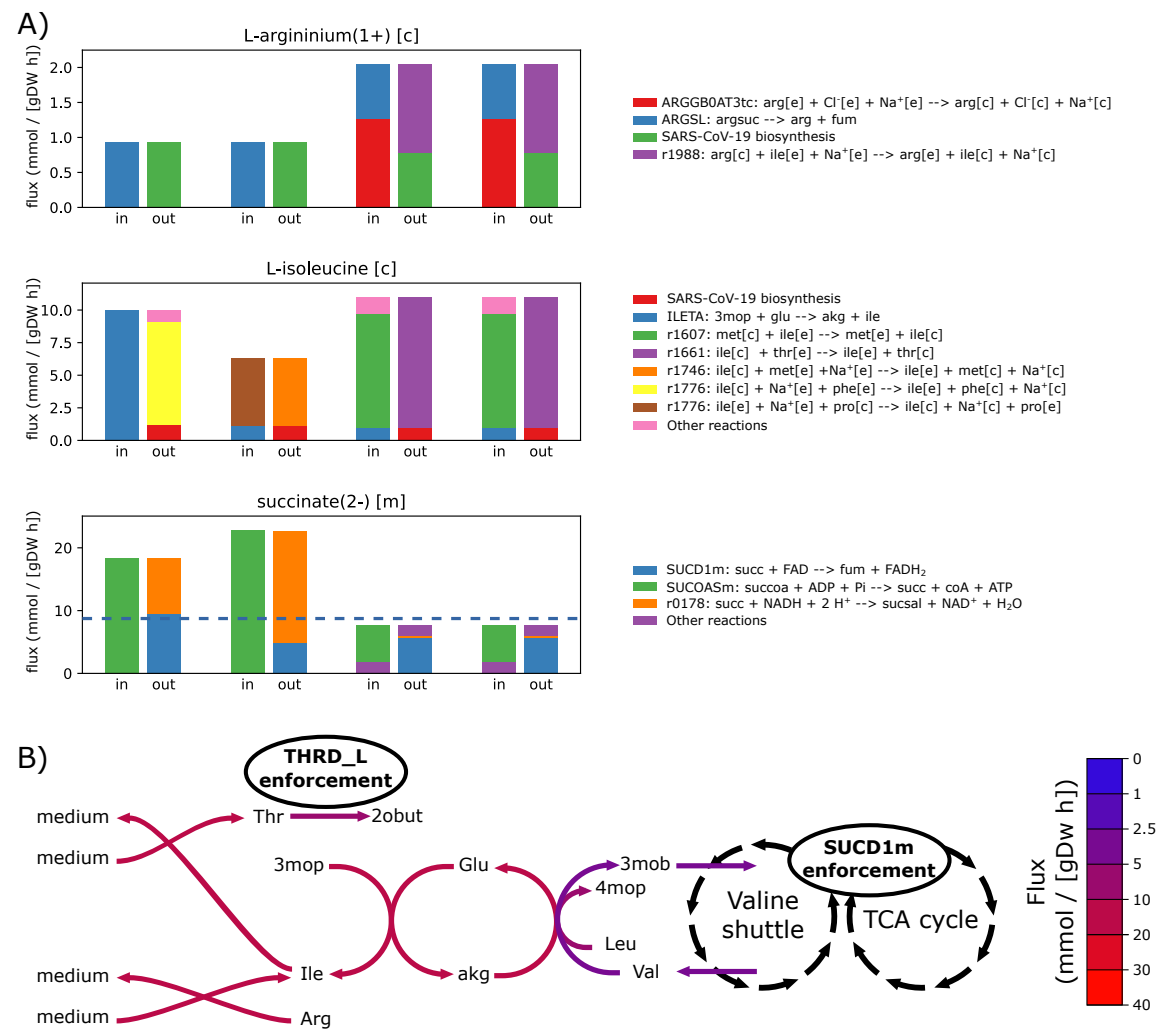

Figure 4 . Effect of the flux enforcement of threonine deaminase (THRD) and succinate dehydrogenase (SUCD1m) reactions on the optimal flux distribution for SARS-CoV-2 production. The enforced flux boundaries were $[8.94 ; 9.14]$ and $\left.[-0.58 ; 8.60] \mathrm{mmol}^{2}\right] \mathrm{gDW}^{-1}[?] \mathrm{h}^{-1}$ for THRD and SUCD1m respectively. (A) Distribution of the producing ('in') and consuming ('out') fluxes for arginine and isoleucine in the cytosol and succinate in the mitochondrial matrix. For each metabolite, the in and out fluxes have been computed in four different conditions; from left to right; unmodified model, flux enforcement of SUCD1m only, flux enforce- 
ment of THRD only, and flux enforcement of both SUCD1m and THRD. On the succinate panel, the dashed blue line corresponds to the upper boundaries to which SUCD1m is enforced $\left.\left(8.60 \mathrm{mmol}^{2}\right] \mathrm{gDW}^{-1}[?] \mathrm{h}^{-1}\right)$. (B)Cartoon representation of the reaction network involving threonine and other metabolites, with reaction fluxes from normal and perturbation conditions colour-mapped onto reaction arrows. Metabolite notations used are: 2-oxobutanoate (2obut), 3-methyl-2-oxobutanoate (3mob), 4-methyl-2-oxopentanoate (4mop), and alpha-keto-glutarate $(\mathrm{akg})$.

\section{SUPPLEMENTARY FIGURES AND FILES}

A)

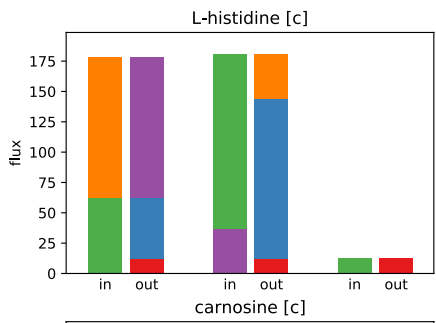

- SARS-COV-19 biosynthesis

NBAHH_ir: carn + $\mathrm{H}_{2} \mathrm{O} \rightarrow \mathrm{NH}+\beta-\mathrm{H}_{4}$

HIStN1: His[e] + $\mathrm{H}^{+}[\mathrm{c}]+2 \mathrm{Na}^{+}[\mathrm{ee}]-->\mathrm{His}[\mathrm{c}]+\mathrm{H}^{+}[\mathrm{e}]+2 \mathrm{Na}^{+}[\mathrm{c}]$

Other reactions (Histidine tradings)

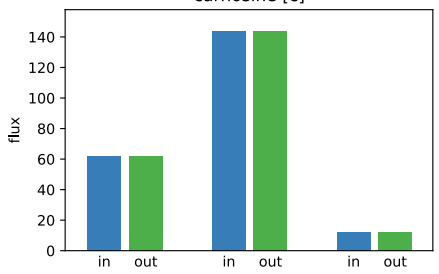

- CARPEPTItc: carn[e] $+\mathrm{H}^{+}[\mathrm{e}]-->\operatorname{carn}[\mathrm{c}]+\mathrm{H}^{+}[\mathrm{c}]$

CARPEPT1tc: carn[e] $+\mathrm{H}^{+}[\mathrm{e}]-\cdots>$ carn $[\mathrm{c}]+$

B)

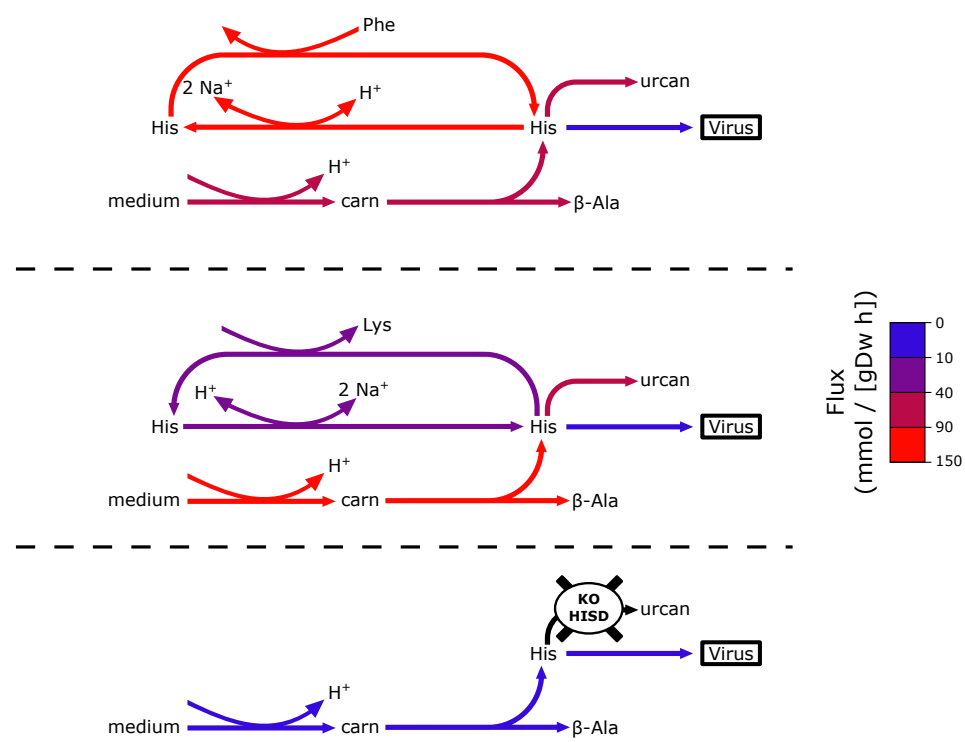

Supplementary Figure 1. Effect of the double knockout of histidase (HISD) and citrate synthase (CSm) on the optimal flux distribution for virus production under minimal media conditions with uptake fluxes set to $-1000 \mathrm{mmol}[?] \mathrm{gDW}^{-1}[?] \mathrm{h}^{-1}$. (A)Distribution of the producing ('in') and consuming ('out') fluxes for histidine and its precursors carnosine in the cytosol. Note that carnosine is only used for the production of histidine in the model histidine is only involved in the SARS-CoV-2 biosynthesis and the HISD reactions (as shown in part (B)). For each metabolite, the in and out fluxes have been computed for the unmodified model (left), the model with CSm reaction blocked (center), and the model with HISD reaction blocked (right). The fluxes in the case of both reactions blocked is not shown, as fluxes in this case are all zero. 
(B) Cartoon representation of the reaction network involving histidine and other metabolites, with reaction fluxes from normal and perturbation conditions colour-mapped onto reaction arrows. The upper panel represents the flux distribution in the normal condition, the center panel represents flux distribution under CSm knockout, and the lower panel represents flux distribution under HISD knockout. Metabolite notations used are: trans-urocanate (urcan), carnosine (carn), $\beta$-alanine ( $\beta$-Ala). Note that when CSm is blocked (middle panel) the flux through the carnosine-to-histidine (the CARNPEPT1tc and NBAHH_ir reactions) increases. This is because the blocking of CSm perturbs proton balances and requires a higher flux through the CARNPEPT1tc reaction, which imports a proton. When HISD reaction is blocked (lower panel), however, the CARNPEPT1tc reaction cannot carry high flux. This is because the only output to histidine is SARS-CoV-2 biomass production, which is limited by other building blocks. Thus, the combined blocking of CSm and HISD reactions (or any other combination of CSm with reactions in the histidine consumption pathway) creates an incompatible situation for proton balancing in the model (i.e. cell).

Supplementary File 1. Results of the flux variability analysis, where flux range for each reaction is calculated while maintaining an optimal biomass flux to SARS-CoV-2 production. Results for simulations with minimal media (flux limits set to -10 or $-1000 \mathrm{mmol}[?] \mathrm{gDW}^{-1}\left[? \mathrm{~h}^{-1}\right.$ ) or rich media are shown. Reactions are ordered according to metabolic subsystems used in the RECON2.2 model, with associated genes identified in the model curation process included as well.

\section{Hosted file}

delattre_etal_S1.xlsx available at https://authorea.com/users/320517/articles/450169inhibiting-the-reproduction-of-covid-19-causing-sars-cov-2-through-perturbations-inhuman-cell-metabolic-network

Supplementary File 2. Results of the single and combined reaction perturbations (gene knock-out simulations) on SARS-CoV-2 production, as performed on all reactions identified as flux-carrying in the flux variability analysis. Simulation results with the minimal media with two different uptake flux limits are shown (flux limits set to -10 or $-1000 \mathrm{mmol}[?] \mathrm{gDW}^{-1}[?] \mathrm{h}^{-1}$ ). Effects of knock-outs are shown as percentage of optimal flux.

\section{Hosted file}

delattre_etal_S2.xlsx available at https://authorea.com/users/320517/articles/450169inhibiting-the-reproduction-of-covid-19-causing-sars-cov-2-through-perturbations-inhuman-cell-metabolic-network

Supplementary File 3. The composition and stoichiometries of building block metabolites in the virus biomass and human lung cell specific maintenance pseudo reactions.

\section{Hosted file}

delattre_etal_S3.xlsx available at https://authorea.com/users/320517/articles/450169inhibiting-the-reproduction-of-covid-19-causing-sars-cov-2-through-perturbations-inhuman-cell-metabolic-network

Supplementary File 4. Results of the flux variability analysis, where flux range for each reaction is calculated while maintaining an optimal flux on the SARS-CoV-2 biomass or human lung cell specific maintenance pseudo reactions. Results for simulations with minimal media (flux limits set to -10 or -1000 $\left.\mathrm{mmol}[?] \mathrm{gDW}^{-1}[?] \mathrm{h}^{-1}\right)$ are shown.

\section{Hosted file}


delattre_etal_S4.xlsx available at https://authorea.com/users/320517/articles/450169inhibiting-the-reproduction-of-covid-19-causing-sars-cov-2-through-perturbations-inhuman-cell-metabolic-network

Supplementary File 5. Results of the single and combined reaction perturbations (gene knock-out simulations) on SARS-CoV-2 biomass and human lung cell specific maintenance pseudo reactions. Reaction pairs, whose combined perturbation results in a virus optimal biomass reduction below $80 \%$ of the original are shown. Simulation results with the minimal media with two different uptake flux limits are shown (flux limits set to -10 or $\left.-1000 \mathrm{mmol}[?] \mathrm{gDW}^{-1}[?] \mathrm{h}^{-1}\right)$.

\section{Hosted file}

delattre_etal_S5.xlsx available at https://authorea.com/users/320517/articles/450169inhibiting-the-reproduction-of-covid-19-causing-sars-cov-2-through-perturbations-inhuman-cell-metabolic-network

Supplementary File 6. The composition of the minimal media used in this study.

\section{Hosted file}

delattre_etal_S6.tsv available at https://authorea.com/users/320517/articles/450169inhibiting-the-reproduction-of-covid-19-causing-sars-cov-2-through-perturbations-inhuman-cell-metabolic-network

\section{AUTHOR CONTRIBUTIONS}

O.S.S. designed the overall study and contributed to model development and analysis. H.D designed the model, implemented it and performed model analyses. K.S contributed in the design and development of additional analysis and simulation tools. All authors contributed to the writing of the manuscript and have given approval to the final version.

\section{ACKNOWLEDGEMENTS}

We thank members of the Soyer group for insightful discussions on model development and analysis. We acknowledge the support of our families, who have endured us during this computational study conducted under COVID-19-enforced lock down and home-working conditions.

\section{DATA ACCESSIBILITY}

All relevant data is presented in the main and supplementary texts.

\section{CODE AVAILABILITY}

Models and their source code are available on O.S.S research group web pages.

\section{FUNDING STATEMENT}

This work was funded by The University of Warwick and by the Biotechnological and Biological Sciences Research Council (BBSRC), with grant no. BB/T010150/1 (to O.S.S.) and no. BB/S506783/1 (to the University of Warwick).

\section{REFERENCES}

1. Aller, S., Scott, A., Sarkar-Tyson, M., \& Soyer, O. S. (2018). Integrated human-virus metabolic stoichiometric modelling predicts host-based antiviral targets against Chikungunya, Dengue and Zika viruses. Journal of the Royal Society Interface . https://doi.org/10.1098/rsif.2018.0125

2. Bar-On, Y. M., Flamholz, A., Phillips, R., \& Milo, R. (2020). Sars-cov-2 (Covid-19) by the numbers. ELife . https://doi.org/10.7554/eLife.57309 
3. Bárcena, M., Oostergetel, G. T., Bartelink, W., Faas, F. G. A., Verkleij, A., Rottier, P. J. M., ... Bosch, B. J. (2009). Cryo-electron tomography of mouse hepatitis virus: Insights into the structure of the coronavirion. Proceedings of the National Academy of Sciences of the United States of America. https://doi.org/10.1073/pnas.0805270106

4. Berzin, V., Rosenthal, G., \& Gren, E. J. (1974). Cellular Macromolecule Synthesis in Escherichia coli Infected with Bacteriophage MS2. European Journal of Biochemistry . https://doi.org/10.1111/j.14321033.1974.tb03547.x

5. Birch, E. W., Ruggero, N. A., \& Covert, M. W. (2012). Determining host metabolic limitations on viral replication via integrated modeling and experimental perturbation. PLoS Comput Biol ,8 (10), e1002746. https://doi.org/10.1371/journal.pcbi.1002746

6. Bordbar, A., Lewis, N. E., Schellenberger, J., Palsson, B. Ø., \& Jamshidi, N. (2010). Insight into human alveolar macrophage and M. tuberculosis interactions via metabolic reconstructions. Mol Syst Biol , 6 , 422. https://doi.org/10.1038/msb.2010.68

7. Bordbar, A., Monk, J. M., King, Z. A., \& Palsson, B. O. (2014). Constraint-based models predict metabolic and associated cellular functions. Nature Reviews Genetics . https://doi.org/10.1038/nrg3643

8. Carinhas, N., Bernal, V., Monteiro, F., Carrondo, M. J. T., Oliveira, R., \& Alves, P. M. (2010). Improving baculovirus production at high cell density through manipulation of energy metabolism. Metabolic Engineering . https://doi.org/10.1016/j.ymben.2009.08.008

9. Chindelevitch, L., Trigg, J., Regev, A., \& Berger, B. (2015). Reply to "Do genome-scale models need exact solvers or clearer standards?" Molecular Systems Biology . https://doi.org/10.15252/msb.20156548

10. Dijkstra, E. W. (1959). A note on two problems in connexion with graphs. Numerische Mathematik, $1(1), 269-271$.

11. Edwards, J. S., \& Palsson, B. O. (2000). The Escherichia coli MG1655 in silico metabolic genotype: Its definition, characteristics, and capabilities. Proceedings of the National Academy of Sciences of the United States of America . https://doi.org/10.1073/pnas.97.10.5528

12. El-Bacha, T., Menezes, M. M. T., Azevedo Silva, M. C., Sola-Penna, M., \& Da Poian, A. T. (2004). Mayaro virus infection alters glucose metabolism in cultured cells through activation of the enzyme 6-phosphofructo 1-kinase. Molecular and Cellular Biochemistry . https://doi.org/10.1023/B:MCBI.0000049154.17866.00

13. Endy, D., Kong, D., \& Yin, J. (1997). Intracellular kinetics of a growing virus: A genetically structured simulation for bacteriophage T7. Biotechnology and Bioengineering .

14. Gille, C., Bolling, C., Hoppe, A., Bulik, S., Hoffmann, S., Hubner, K., ... Holzhutter, H. G. (2010). HepatoNet1: A comprehensive metabolic reconstruction of the human hepatocyte for the analysis of liver physiology. Molecular Systems Biology . https://doi.org/10.1038/msb.2010.62

15. Ibarra, R. U., Edwards, J. S., \& Palsson, B. O. (2002). Escherichia coli K-12 undergoes adaptive evolution to achieve in silico predicted optimal growth. Nature . https://doi.org/10.1038/nature01149

16. Ikeda, M., \& Kato, N. (2007). Modulation of host metabolism as a target of new antivirals. Advanced Drug Delivery Reviews . https://doi.org/10.1016/j.addr.2007.03.021

17. Jain, R., \& Srivastava, R. (2009). Metabolic investigation of host/pathogen interaction using MS2infected Escherichia coli.BMC Systems Biology . https://doi.org/10.1186/1752-0509-3-121

18. Leyssen, P., De Clercq, E., \& Neyts, J. (2008). Molecular strategies to inhibit the replication of RNA viruses. Antiviral Research . https://doi.org/10.1016/j.antiviral.2008.01.004

19. Mahmoudabadi, G., Milo, R., \& Phillips, R. (2017). Energetic cost of building a virus. Proceedings of the National Academy of Sciences of the United States of America. https://doi.org/10.1073/pnas.1701670114

20. Mardinoglu, A., Agren, R., Kampf, C., Asplund, A., Uhlen, M., \& Nielsen, J. (2014). Genome-scale metabolic modelling of hepatocytes reveals serine deficiency in patients with non-alcoholic fatty liver disease. Nature Communications . https://doi.org/10.1038/ncomms4083

21. Maynard, N. D., Gutschow, M. V, Birch, E. W., \& Covert, M. W. (2010). The virus as metabolic engineer. Biotechnol J , 5 (7), 686-694. https://doi.org/10.1002/biot.201000080

22. Maynard, N. D., Birch, E. W., Sanghvi, J. C., Chen, L., Gutschow, M. V, \& Covert, M. W. 
(2010). A forward-genetic screen and dynamic analysis of lambda phage host-dependencies reveals an extensive interaction network and a new anti-viral strategy. PLoS Genet ,6 (7), e1001017. https://doi.org/10.1371/journal.pgen.1001017

23. Munger, J., Bajad, S. U., Coller, H. A., Shenk, T., \& Rabinowitz, J. D. (2006). Dynamics of the cellular metabolome during human cytomegalovirus infection. PLoS Pathogens . https://doi.org/10.1371/journal.ppat.0020132

24. Munger, J., Bennett, B. D., Parikh, A., Feng, X. J., McArdle, J., Rabitz, H. A., .. Rabinowitz, J. D. (2008). Systems-level metabolic flux profiling identifies fatty acid synthesis as a target for antiviral therapy. Nature Biotechnology . https://doi.org/10.1038/nbt.1500

25. Neuman, B. W., Adair, B. D., Yoshioka, C., Quispe, J. D., Orca, G., Kuhn, P., .. Buchmeier, M. J. (2006). Supramolecular Architecture of Severe Acute Respiratory Syndrome Coronavirus Revealed by Electron Cryomicroscopy. Journal of Virology . https://doi.org/10.1128/jvi.00645-06

26. Neuman, B. W., Joseph, J. S., Saikatendu, K. S., Serrano, P., Chatterjee, A., Johnson, M. A., ... Kuhn, P. (2008). Proteomics Analysis Unravels the Functional Repertoire of Coronavirus Nonstructural Protein 3. Journal of Virology . https://doi.org/10.1128/jvi.02631-07

27. Neuman, B. W., Kiss, G., Kunding, A. H., Bhella, D., Baksh, M. F., Connelly, S., ... Buchmeier, M. J. (2011). A structural analysis of M protein in coronavirus assembly and morphology. Journal of Structural Biology . https://doi.org/10.1016/j.jsb.2010.11.021

28. Papp, B., Pal, C., \& Hurst, L. D. (2004). Metabolic network analysis of the causes and evolution of enzyme dispensability in yeast.Nature , 429 (6992), 661-664. https://doi.org/10.1038/nature02636

29. Quek, L. E., Dietmair, S., Hanscho, M., Martinez, V. S., Borth, N., Nielsen, L. K. (2014) Reducing Recon 2 for steady-state flux analysis of HEK cell culture. Journal of Biotechnology 184. doi: 10.1016/j.jbiotec.2014.05.021

30. Raman, K., \& Chandra, N. (2009). Flux balance analysis of biological systems: Applications and challenges. Briefings in Bioinformatics . https://doi.org/10.1093/bib/bbp011

31. Robinson, J. L., Kocabaş, P., Wang, H., Cholley, P. E., Cook, D., Nilsson, A., .. Nielsen, J. (2020). An atlas of human metabolism. Science Signaling . https://doi.org/10.1126/scisignal.aaz1482

32. Segrè, D., Vitkup, D., \& Church, G. M. (2002). Analysis of optimality in natural and perturbed metabolic networks. Proc Natl Acad Sci U S A , 99 (23), 15112-15117. https://doi.org/10.1073/pnas.232349399

33. Senior, N. J., Sasidharan, K., Saint, R. J., Scott, A. E., Sarkar-Tyson, M., Ireland, P. M., ... Titball, R. W. (2017). An integrated computational-experimental approach reveals Yersinia pestis genes essential across a narrow or a broad range of environmental conditions. BMC Microbiology . https://doi.org/10.1186/s12866-017-1073-8

34. Schuster, S., Pfeiffer, T., \& Fell, D. A. (2008). Is maximization of molar yield in metabolic networks favoured by evolution? J Theor Biol, 252 (3), 497-504. https://doi.org/10.1016/j.jtbi.2007.12.008

35. Simmonds, P., Aiewsakun, P., \& Katzourakis, A. (2019). Prisoners of war - host adaptation and its constraints on virus evolution. Nature Reviews Microbiology . https://doi.org/10.1038/s41579-0180120-2

36. Swainston, N., Smallbone, K., Hefzi, H., Dobson, P. D., Brewer, J., Hanscho, M., ... Mendes, P. (2016). Recon 2.2: from reconstruction to model of human metabolism. Metabolomics . https://doi.org/10.1007/s11306-016-1051-4

37. Thiele, I., Swainston, N., Fleming, R. M. T., Hoppe, A., Sahoo, S., Aurich, M. K., .. Palsson, B. O. (2013). A community-driven global reconstruction of human metabolism. Nature Biotechnology . https://doi.org/10.1038/nbt.2488

38. Thiele, I., \& Palsson, B. Ø. (2010). A protocol for generating a high-quality genome-scale metabolic reconstruction. Nat Protoc ,5 (1), 93-121. https://doi.org/10.1038/nprot.2009.203

39. Uhlén, M., Fagerberg, L., Hallström, B. M., Lindskog, C., Oksvold, P., Mardinoglu, A., .. Pontén, F. (2015). Tissue-based map of the human proteome. Science . https://doi.org/10.1126/science.1260419

40. Waldbauer, J. R., Coleman, M. L., Rizzo, A. I., Campbell, K. L., Lotus, J., \& Zhang, L. (2019). Nitrogen sourcing during viral infection of marine cyanobacteria. Proceedings of the National Academy of Sciences of the United States of America . https://doi.org/10.1073/pnas.1901856116 
41. Wang, Q.-Y., Bushell, S., Qing, M., Xu, H. Y., Bonavia, A., Nunes, S., .. Shi, P.-Y. (2011). Inhibition of Dengue Virus through Suppression of Host Pyrimidine Biosynthesis. Journal of Virology . https://doi.org/10.1128/jvi.02510-10

42. Wishart, D. S., Feunang, Y. D., Guo, A. C., Lo, E. J., Marcu, A., Grant, J. R., ... Wilson, M. (2018). DrugBank 5.0: A major update to the DrugBank database for 2018. Nucleic Acids Research . https://doi.org/10.1093/nar/gkx1037

43. Wong, E. H. M., Smith, D. K., Rabadan, R., Peiris, M., \& Poon, L. L. M. (2010). Codon usage bias and the evolution of influenza A viruses. Codon Usage Biases of Influenza Virus. BMC Evolutionary Biology . https://doi.org/10.1186/1471-2148-10-253

44. Yates, A. D., Achuthan, P., Akanni, W., Allen, J., Allen, J., Alvarez-Jarreta, J., .. Flicek, P. (2020). Ensembl 2020.Nucleic Acids Research . https://doi.org/10.1093/nar/gkz966

45. Yin, J., \& Redovich, J. (2018). Kinetic Modeling of Virus Growth in Cells. Microbiology and Molecular Biology Reviews . https://doi.org/10.1128/mmbr.00066-17

46. You, L., Suthers, P. F., \& Yin, J. (2002). Effects of Escherichia coli physiology on growth of phage T7 in vivo and in silico. Journal of Bacteriology . https://doi.org/10.1128/JB.184.7.1888-1894.2002

47. Yu, Y., Clippinger, A. J., \& Alwine, J. C. (2011). Viral effects on metabolism: Changes in glucose and glutamine utilization during human cytomegalovirus infection. Trends in Microbiology . https://doi.org/10.1016/j.tim.2011.04.002 